\title{
La Convention nationale au miroir des Archives Parlementaires
}

The National Convention as reflected in the Archives Parlementaires

Corinne Gomez-Le Chevanton et Françoise Brunel

\section{(2) OpenEdition \\ 1 Journals}

Édition électronique

URL : https://journals.openedition.org/ahrf/13604

DOI : $10.4000 /$ ahrf. 13604

ISSN : 1952-403X

Éditeur :

Armand Colin, Société des études robespierristes

Édition imprimée

Date de publication : 1 septembre 2015

Pagination : 11-29

ISBN : 9782200929855

ISSN : 0003-4436

Référence électronique

Corinne Gomez-Le Chevanton et Françoise Brunel, «La Convention nationale au miroir des Archives Parlementaires », Annales historiques de la Révolution française [En ligne], 381 | juillet-septembre 2015, mis en ligne le 01 septembre 2018, consulté le 22 avril 2022. URL : http://journals.openedition.org/ ahrf/13604 ; DOI : https://doi.org/10.4000/ahrf.13604 
ARTICLES

\section{LA CONVENTION NATIONALE AU MIROIR DES ARCHIVES PARLEMENTAIRES}

Corinne GOMEZ-LE CHEVANTON et Françoise BRUNEL

Les auteures, éditrices des Archives Parlementaires (Première série), présentent l'histoire mouvementée de cette collection fondée il y a 150 ans. À l'heure de la numérisation des grands corpus documentaires, elles soulignent les « moments » très diversifiés de cette source construite par des éditeurs, d'abord bibliothécaires ou fonctionnaires de la Chambre des députés, puis historiens de la Révolution française, soucieux de dénombrement et de cohérence sérielle. II s'agit aussi de montrer comment la « colonne vertébrale » de cette construction, le procès-verbal, est d'une fiabilité douteuse à partir du début de thermidor an II. L'analyse de la construction postthermidorienne jusqu'au tournant de brumaire-frimaire an III, marqué par l'exceptionnalité de fait du procès du représentant Carrier, pose alors un vrai problème épistémologique, dans des constructions politiques orientées.

Mots-clés : Archives Parlementaires, Convention nationale, édition de sources, moment thermidorien, procès Carrier

Singulière histoire que celle de la publication du grand corpus documentaire des Archives Parlementaires, entamée il y a un siècle et demi et encore inachevée. C'est en 1862 que le Corps législatif, probablement «à l'initiative libérale de M. le duc de Morny » son président, inaugure la publication d'un Recueil complet des débats législatifs et politiques des Chambres françaises de 1800 à 1860, faisant suite à la réimpression de l'ancien "Moniteur » et comprenant grand nombre de documents 
inédits ${ }^{1}$. Ainsi se constitue ce qui devient, en 1867, la « Deuxième série » des Archives parlementaires ${ }^{2}$. À cette date, les mêmes Jérôme Mavidal et Émile Laurent, sous-bibliothécaires au Corps législatif, sont en effet chargés d'un « travail partiel » : fournir une « introduction au Moniteur ». Le Moniteur, expliquent-ils, ayant le mérite de donner la parole aux " témoins oculaires », " en présence des témoins intéressés de tous les partis », ne parut que le 24 novembre 1789 et « il ne manquait à ce recueil que d'avoir commencé avec la Révolution ; car [...] c'est dans l'histoire des révolutions qu'il importe de ne rien omettre de ce qui tend à faire connaître leurs premiers mouvements, leurs causes, les symptômes qui les annoncèrent et à nous initier [...] dans la génération de ces importants phénomènes qui ont en naissant la force de tout détruire $»^{3}$. Si, en effet, la décision prise par le Corps législatif en 1862 peut s'expliquer tant par le souci de donner en « un format plus commode que le Moniteur » (in folio) « le compte rendu absolument exact des débats législatifs et le texte définitif des lois adoptées $»^{4}$, que par le rôle politique de Morny, soucieux d'apaiser les tensions intérieures et d'encourager un élan patriotique à l'orée de l'aventure mexicaine, les motivations de 1867 sont plus obscures : Morny est mort en 1865 et ne peut être « l'incitateur » direct ; en revanche, depuis 1863, le Corps législatif compte une trentaine d'opposants dotés de fortes personnalités : Adolphe Thiers, mais aussi les républicains Jules Favre ou Émile Ollivier. Face aux menaces qui s'annoncent, alors que l'empereur doit rendre le droit d'interpellation aux députés, n'est-il pas judicieux d'inscrire le régime dans le sillage ouvert par la Révolution de 1789 ? L' « Avant-propos » de nos zélés bibliothécaires porte la marque de cet espoir, lorsqu'ils écrivent, dans leur éloge du système représentatif : «C'est dans une assemblée homogène, composée de députés temporaires du peuple, qu'on trouvait enfin les plus sûrs moyens de faire prédominer,

(1) La formule concernant le rôle de Morny est de Mavidal et Laurent dans leur «Avertissement» au premier volume, mais ne figure pas dans la publication des Cahiers des États généraux... (Extrait des Archives Parlementaires), tome I, Paris, Paul Dupont, 1868. Elle disparaît évidemment de la seconde édition de 1879. Voir Armand BRETTE, « Les cahiers de doléances et les Archives Parlementaires », La Révolution française, tome 47, juillet-décembre 1904, p. 5-27.

(2) Le tome IX (du $1^{\text {er }}$ janvier 1806 au 15 septembre 1815) porte le titre Archives Parlementaires de 1787 à 1860. Recueil complet des débats législatifs et politiques des Chambres françaises... sous la direction de MM. Jules (sic pour Jérôme) Mavidal et Émile Laurent, Deuxième série (1800 à 1860), Paris, 1867.

(3) Cahiers des États généraux..., op. cit., « Avant-Propos », p. 1.

(4) La publication de la «Deuxième série » atteint le tome XVII ( $1^{\mathrm{er}}$ avril au 30 décembre 1816) en 1870. Aucune explication méthodologique n'est donnée avant 1902 et le tome CV (7 juin 1836 au 4 juillet 1836), qui précise dans une «note » que la méthode « est la même que celle indiquée par nous dans la note imprimée en tête du tome LXI de la $1^{\text {re }}$ série ». 
par l'intérêt même des hommes qui la composaient, le bien public sur les passions particulières $»^{5}$.

Sans nier la place de choix parmi les sources du $\mathrm{XIX}^{\mathrm{e}}$ siècle de la «Deuxième série » des Archives Parlementaires, dont témoigne l'ouvrage consacré à La Chambre des députés en 1837-1839, entremêlant prosopographie, histoire politique et « technique »du fonctionnement de l'Assemblée et étude linguistique des débats autour de l'Adresse de $1839^{6}$, il faut constater que la série semble s'éteindre, dans une indifférence assez sidérante, avant même la déclaration de la Grande Guerre : le dernier volume, le tome CXXVI (17 juin 1839 au 8 juillet 1839) parait en 1912 et il y a peu de chance que cette « série » parvienne au terme initialement fixé de 1860.

Mais c'est évidemment la puînée, devenue « Première série (1787 à 1799) » des Archives Parlementaires de 1787 à 1860, que nous voulons évoquer, son histoire mouvementée et fragmentée, mais toujours vivante en 2015, phénomène assez étonnant dans les institutions scientifiques pour être souligné. L'histoire est mouvementée car la publication est née sans réelle préparation pour une documentation de fait vertigineuse, dont Mavidal et Laurent, bibliothécaires, mais non historiens et étrangers à la recherche historique même de leur époque, semblent avoir négligé les écueils. Pourtant, la carrière administrative et politique de Mavidal (1825-1896) décolle avec l'avènement de la République et, en 1875, on le retrouve " Chef du Bureau de l'expédition des Lois et des procès-verbaux », chevalier de la Légion d'honneur et directeur de la publication du tome VII (5 mai au 15 septembre 1789) des Archives Parlementaires ${ }^{7}$. Émile Laurent (1819-1897), venu à Paris pour se faire un nom par la plume, a publié, sous les pseudonymes de Georges d'Harmonville et surtout d'Émile Colombey, des romans, éditions critiques ou essais littéraires, dont, en 1858, Ninon de Lenclos et sa cour. L'historien perce alors, peut-être, sous le publiciste, mais se trouve tout de même éloigné de la rigueur scientifique d'une publication de sources innombrables, parfois difficilement authentifiables. Le défi, surtout, de commencer par la publication des « cahiers de doléances » était

(5) Cahiers des États généraux..., op. cit., « Avant-Propos », p. 15.

(6) Voir Louis GIRARD, William SERMAN, Édouard CADET, Rémi GossEz, La Chambre des députés en 1837-1839. Composition, activité, vocabulaire, Paris, Publications de la Sorbonne, 1976.

(7) Voir tome VIII, Archives Parlementaires..., première série, sous la direction de M. J. MAVIDAL et de MM. É. LAURENT et É. ClAVEL, sous-bibliothécaires de l'Assemblée nationale, Paris, 1875. Et sur les attributions de chacun dans l'administration de la Chambre des députés, voir Almanach national. Annuaire officiel de la République française pour 1876, présenté au Président de la République, Paris, Berger-Levrault, 1876, p. 96-97. 
considérable : sans méthode et sans information sur les sources ${ }^{8}$, les auteurs allaient au-devant des critiques les plus sévères. Mais, force est de constater que la collection poursuivit son chemin et qu' après une interruption de plus de quarante ans, elle atteint en 2012 le cent deuxième volume, les tomes CIII et CIV étant en préparation.

Cette source « construite » a donc résisté au temps, mais en évoluant évidemment très largement au fil du siècle et demi de son existence, en fonction des méthodes, des exigences tant des éditeurs que des lecteurs, historiens, juristes, politistes ou linguistes, tous spécialistes d'histoire de la Révolution française. C'est ce que nous tenterons d'illustrer, pour la seule période de la Convention nationale que couvrent actuellement les tomes LII (22 septembre au 26 octobre 1792), paru en 1897, au tome CII (2012), qui nous conduit au 12 frimaire an III (2 décembre 1794). Dans cette série, construite et édifiée par les éditeurs scientifiques sur des documents rédigés, filtrés, triés par les pouvoirs, se laisse entendre toutefois l'écho des débats et combats de l'Assemblée fondatrice de notre République, mais aussi l'une des meilleures caisses de résonance d'une opinion devenue véritablement nationale.

\section{Les aventures des Archives Parlementaires : une histoire savante dans les vents, d'une Exposition universelle (Paris, 1867) à l'autre (Paris, 2025)?}

Avant d'aborder l'histoire de la Convention nationale que retracent les Archives Parlementaires au fil des cinquante et un volumes aujourd'hui publiés, évoquons cette « Première série » dont nous allons radiographier la moitié des volumes et dont il faut situer les heurs et malheurs, car elle est la rescapée d'une entreprise extraordinaire de « communication » impériale, poursuivie judicieusement par les Républiques (la III ${ }^{\mathrm{e}}$, puis la $\mathrm{V}^{\mathrm{e}}$, tout au moins). Une publication sur la période «1787-1860», quand nous constatons que vingt-six mois de l'entreprise (22 septembre 17922 décembre 1794) couvrent cinquante et un volumes, plus de 300000 pages ! L'ampleur de la tâche semble démesurée, vaine et finalement sans grand intérêt scientifique : « tout cela pour ça ? » interrogeront à juste titre les spécialistes des larges séries de la longue durée ou des World Studies. Certes, jusque dans une présentation toujours désuète (par colonne), mais

(8) Mavidal et Laurent semblent ignorer la célèbre «Collection Camus » des Archives nationales, les cartons et registres consacrés aux États généraux et réunis dès 1790, BA 1 à 91 et BIII 1 à 174. Voir, ci-dessous, la critique d'Armand Brette en 1904. 
dont nul ne peut nier l'évolution de la numérotation et présentation des notes, de la typographie générale (suppression des exposants multiples, des abréviations paléographiques, des esperluettes et autres caractères décoratifs inutiles, heurtant la fluidité de la lecture), des polices de caractères, depuis vingt ans, la publication des Archives Parlementaires peut, pour les garants des fonds publics, constituer un «conservatoire » de pratiques défuntes d'une érudition un brin cocardière. Il n'en est évidemment rien, à nos yeux, et l'hypercritique nous permettra de rendre justice à un travail séculaire qui, évidemment, ne constitue en rien une série homogène. Il ne faudrait pas, en effet, que les historiens contemporains, pris au piège de la numérisation et, nous en convenons, d'atours esthétiques peu évolutifs, transforment en « une » source, plusieurs moments différenciés et authentifiables de sources ayant pour objet les « débats législatifs et politiques... », au sein desquels la Convention, actuellement encore en traitement, a elle-même déjà connu quatre « moments $»^{9}$.

Le «premier moment» de la «Première série» des Archives Parlementaires est non défini par les fondateurs de la collection : Mavidal et Laurent, en lançant leur titanesque entreprise, n'ont jamais fixé de normes scientifiques ou construit une méthode d'édition des textes. Heureux temps d'un libertarianisme intellectuel ? Il semble que ce soit plutôt, hélas, ignorance et sous-estimation du travail de publication des sources. Sans nous appesantir sur les six premiers volumes (1867-1870), dont le rythme de parution est tellement intense qu'il impressionne, mais étonne aussi les éditrices contemporaines, arrêtons-nous sur le tome VIII, publié en $1875^{10}$. Dans un article de 1904, Armand Brette, très sérieux érudit et membre de la récente «Commission» d'histoire économique de la Révolution

(9) Il nous semble que le pourtant très perspicace historien américain de la Révolution française, Timothy Tackett, n'a pas toujours échappé à ce travers. Voir Timothy TACKETT et Nicolas DÉPLANCHE, «L'idée du "complot" dans l'œuvre de Georges Lefebvre : une remise en cause à partir d'une nouvelle source », La Révolution française [En ligne], Georges Lefebvre, mis en ligne le 5 juillet 2010. URL : http:///rf.revues.org/171. Timothy Tackett avait expérimenté la construction de son « indice de sensibilité aux complots» en traitant un échantillon de tomes des Archives Parlementaires, alors en numérisation par un projet « Google Livres » (automne 2008-printemps 2009). Le projet a été réalisé par les Archives numériques de la Révolution française, bibliothèques de l'université de Stanford et Bibliothèque nationale de France : http://frda.stanford.edu:fr:ap. On peut regretter l'approximation de la présentation des Archives Parlementaires sur ce site dédié à la recherche ; expliquant que les Archives Parlementaires constituent « un recueil précis des délibérations parlementaires »!, la notice indique qu'a été codé en TEI le texte des tomes parus jusqu'en 1914 mais, nous explique-t-on, « [ces volumes] relatent les événements allant de la convocation des états généraux à la première moitié de la Terreur ».

(10) Archives Parlementaires, Première série, t. VIII, 5 mai 1789 au 15 septembre 1789, Paris, 1875. Ce volume est imprimé par ordre de l'Assemblée nationale, sous la direction de M. J. MAVIDAL [...] et de MM. É. LAURENT et É. CLAVEL. 
française, créée à l'instigation de Jaurès en 1903, écrit : «Ce que l'on peut seulement garder de ce souvenir, c'est la grande hâte qui a présidé à la naissance de cette collection, et qui se manifeste de la manière la plus grave par les singulières imperfections des premiers volumes, on peut même dire de tous ceux qui concernent l'Assemblée constituante $»^{11}$. Brette souligne donc comment la retranscription des débats de l'Assemblée constituante est aussi approximative, voire fautive, que la publication des « cahiers de doléances ». "Les éditeurs des Archives Parlementaires, écritil, n'ont pas été mieux avertis en cette partie » et, faute d'être informés des règlements, confondent « opinions imprimées » et discours prononcés, faisant ainsi discourir à la tribune des députés suppléants qui n'y avaient aucune place. Et que dire de l'attribution au 30 juin 1789 du « début de l'Assemblée nationale »? On peut en convenir : l'édition des volumes des Archives Parlementaires offre peu de fiabilité durant trente ans. Ainsi, écrit Pierre Caron, «la méthode employée par les éditeurs des Archives Parlementaires avait été sévèrement critiquée ; on leur reprochait surtout de combiner arbitrairement avec le texte des procès-verbaux officiels, celui de comptes rendus empruntés à divers journaux et de fabriquer ainsi une sorte de mosaïque qui ne pouvait être utilisée par l'historien qu' au prix de longues et minutieuses vérifications $»^{12}$.

C'est justement avec le premier volume consacré à la Convention nationale, en 1897, que les quatre éditeurs, dégagés sans doute de la tutelle des « fondateurs » et comptant surtout parmi eux un archiviste-paléographe, « véritable » historien, André Ducom (1861-1923), entament, de fait, le « deuxième moment » de la publication de la «Première série » (donc le « premier moment» de la Convention) $)^{13}$. Les auteurs introduisent un ordre logique (et méthodologique) peu perceptible précédemment. Comme ils l'indiquent, ils travaillent « le procès-verbal constamment sous les yeux » et donnent « un compte rendu de la séance aussi complet que possible, à l'aide du Moniteur et de divers autres journaux ». Toutefois, ajoutent-ils, « nous continuons d'introduire à leur ordre, en indiquant les collections où nous les recueillons, les rapports et discours à part in extenso, par ordre de

(11) Armand BRETTE, «Les cahiers de 1789 et les Archives Parlementaires », op. cit., p. 6. Brette est l'éditeur savant du Recueil de documents relatifs à la convocation des États généraux de 1789, Paris, Imprimerie nationale, 5 volumes, 1894-1904. Sur le rôle central de la « Commission Jaurès », voir Christine PEYRARD et Michel VOVELLE (dir.), Héritages de la Révolution française à la lumière de Jaurès, Aix-en-Provence, PUP, 2002.

(12) Pierre CARON, Manuel pratique pour l'étude la Révolution française, Paris, 1912, nouvelle édition mise à jour, Paris, Picard, 1947, p. 205.

(13) Archives Parlementaires, t. LII, du 22 septembre 1792 au 26 octobre 1792, 1897. 
l'Assemblée nationale ou par le soin des auteurs eux-mêmes $»^{14}$. C'est bien là que le bât blesse : toujours sans méthode rigoureuse, la série continue de compiler sans vérification des textes, certes passionnants, mais sans réel rapport avec la publication journalière des séances de l'Assemblée. Un exemple nous semble particulièrement illustratif : la séance du lundi 24 juin 1793 présente le débat sur l'adoption de la Déclaration des droits et de l'Acte constitutionnel. Présidée par Collot d'Herbois, la séance est ouverte à 10 heures du matin et levée à 6 heures et demi du soir : quarante pages sont nécessaires pour retracer les débats et donner les textes adoptés. Or la journée du 24 juin occupe plus de trois cents pages du tome LXVII : d'où vient ce remplissage ? Les éditeurs ont adjoint soixante-deux «Annexes », brochures, opuscules, opinions publiés par des conventionnels, dont Les éléments du républicanisme de Billaud-Varenne qui n'ont rien à voir avec cette date ${ }^{15}$, puisant comme ils le disent dans les imprimés de la Bibliothèque nationale et, surtout, dans la Collection Portiez (de l'Oise) de la Chambre des députés. C'est finalement le tome LXXII, du 11 août 1793 au 24 août 1793, qui marque un tournant méthodologique et ouvre le «troisième moment » des Archives Parlementaires ${ }^{16}$. Rappelant les critiques adressées à la publication conçue par Mavidal et Laurent, les éditeurs disent inaugurer « une nouvelle méthode » pour remplir les vœux « scientifiques » des historiens, en insérant « en gros caractères chacun des paragraphes du procès-verbal », les faisant suivre, en caractères plus petits : $1^{\circ}$ des documents authentiques qui existent soit dans les bibliothèques, soit aux Archives nationales ; $2^{\circ}$ du compte rendu du journal qui semble le plus complet. Ainsi commence à s'établir une hiérarchie raisonnée des éléments constitutifs d'une séance, construite comme un Meccano dont les pièces de base seraient toujours le procès-verbal. Ce «moment » s'achève en 1913, avec la publication du tome LXXXII : la Grande Guerre l'interrompt, mais la « pause » durera près d'un demi-siècle, faisant écrire à Pierre Caron de la collection, non reprise après 1918, qu' « il est douteux qu'elle soit destinée à l'être jamais $»^{17}$.

C'est à l'infatigable persévérance de Georges Lefebvre que les Archives Parlementaires doivent leur renaissance (qu'il ne verra pas, puisqu'il meurt en 1959) : en effet, juste retraité après la Seconde guerre

(14) Ibidem, t. LII, p. 65

(15) Ibid., t. LXVII, du 20 juin 1793 au 30 juin 1793, 1905. Nous avions, par ailleurs, indiqué une probable publication de la brochure de Billaud vers le 15 février 1793.

(16) Ibid., t. LXXII, 1907, « Avertissement ».

(17) Ibid., t. LXXXII, Du 30 frimaire an II au 15 nivôse an II (20 décembre 1793 au 4 janvier 1794), 1913. Voir Pierre CARON, Manuel pratique..., op. cit., p. 205. 
mondiale, le maitre des études révolutionnaires consacre largement son temps à la restructuration et au réveil de la « Commission Jaurès ». Lefebvre sait pouvoir compter sur Édouard Herriot, qu'il connaît depuis 1921 et avec lequel il a directement travaillé pour le $150^{\mathrm{e}}$ anniversaire de la Révolution : en 1951, il donne pour objectif prioritaire à la Commission la reprise du travail sur les Archives Parlementaires. Mais, comme l'indique le Président Herriot, l'Assemblée n'a plus les moyens humains qui étaient les siens au début du siècle ; Georges Lefebvre, toutefois, obtient en 1957 que le CNRS inclut dans son plan quinquennal de recherches la reprise de la publication et débloque des crédits de vacations pour effectuer les dépouillements ${ }^{18}$. En 1961, lorsque parait le tome LXXXIII, la Collection change profondément d'approche du contenu et devient la construction, non plus de bibliothécaires et fonctionnaires de la Chambre des députés, mais celle d'historiens de la Révolution française et, en premier lieu, de Marc Bouloiseau (1907-1999). Dès 1960, Bouloiseau avait émis un avis ironique sur ce qui devenait sa tâche de "maître-assistant de recherche » à « la Sorbonne », comme on disait alors : "Sans être close [écrivait-il] l'ère des Recueils était en nette régression »- depuis les années 1930 - et il n'était plus temps « d'engager à l'aveuglette des fabricants de Recueils $»^{19}$. On ne peut être plus clair, ce que confirme Marcel Reinhard, alors directeur de l'Institut d'Histoire de la Révolution française, dans l'avant-propos qu'il donne au volume : retraçant l'expérience passée, il précise les objectifs d'une collection dorénavant très soucieuse de cohérence sérielle. Le protocole de la publication (toujours en vigueur) est fixé : le procès-verbal de la Convention, imprimé en caractère gras, est dorénavant la colonne vertébrale du montage et la publication des pièces conservées dans la série $\mathrm{C}$ des Archives nationales fournit la chair, tout en gardant l'apport des débats d'Assemblée transmis par les journaux. Les multiples annexes, parfois sans justification, disparaissent, mais la rubrique « Affaires non mentionnées au procès-verbal » remplit pleinement sa fonction, à la date de séance. Enfin, pour une plus grande commodité de lecture, les « affaires » sont numérotées, les pièces classées ${ }^{20}$. Mais la publication présentait toujours, en 1978-1980, les caractéristiques normatives des années 1960, remises en cause par l'harmonisation entreprise à l'École nationale des Chartes : ponctuation ou

(18) Voir Christine PeYRARD et Michel Vovelle (dir.), Héritages..., op. cit., p. 49-51.

(19) Marc BouloiseAU, « De Jaurès à Georges Lefebvre. La Commission d'Histoire économique de la Révolution », Hommage à Georges Lefebvre (1874-1959), Paris, Société des études robespierristes, 1960, p. 61.

(20) Archives Parlementaires, t. LXXXIII, 16 nivôse au 8 pluviôse an II (5 au 27 janvier 1794), Paris, CNRS, 1961, p. VII-IX. 
graphie des pièces littéralement « copiées » ne facilitaient pas une lecture «fluide» des sources ${ }^{21}$. Par ailleurs, la collection conservait encore son aspect initial de « recueil », en intégrant des sources liées, mais exogènes au principe alors défini : « Toute la série $\mathrm{C}$, rien que la série $\mathrm{C}$ » (puisque ce qui est « non-mentionné dans le procès-verbal » comporte à la fois des pièces de la série $\mathrm{C}$ et des affaires effectivement hors procès-verbal pour des raisons évidemment politiques). Le « quatrième moment » de la Première série, le « troisième » de la publication concernant la Convention nationale, que nous appellerons le «moment Bouloiseau » se poursuit avec les volumes publiés sous la responsabilité de Jean-Claude Perrot, puis de Philippe Gut, de 1971 à 1978. D'ailleurs, le tome XCII (1980) n'annonçait que les changements de transcription déjà mentionnés. Mais c'est avec le tome XCIII (21 messidor au 12 thermidor an II) $)^{22}$ qu'est né le moment « actuel », toujours soucieux de plus de précision, « cinquième moment » des Archives Parlementaires et « quatrième » de la publication de la Convention nationale. Certes, depuis les années 1980, les tables chronologiques et les index ont évolué, pour d'évidentes raisons de saisie des documents, mais la contrainte des sources a surtout imposé une approche différente de la publication. En établissant le manuscrit de ce volume, les éditrices (Aline Alquier et Françoise Brunel) constatent une « anomalie »: le procès-verbal de la séance du 2 thermidor est, sans explication, signé de deux députés alors notoirement incarcérés ou clandestins, Henry-Larivière et Laurenceot, tandis que les quatre autres, Bailly, Delecloy, Villers et Delaunay (d'Angers, en fait « le jeune ») avaient peu de chances d'être élus secrétaires à cette date. En effet, la séance du $1^{\text {er }}$ thermidor (au soir) indique le renouvellement (logique) du bureau : Collot est élu président, Le Vasseur (de la Meurthe), Bar, Portiez, Legendre (de Paris), André Dumont et Brival, secrétaires. Il revint à la « responsable de la publication » de trouver une explication et, après d'infructueuses interrogations et recherches, elle découvrit dans le procès-verbal du 3 brumaire an IV, donc à la séparation de la Convention, une loi disposant : «Les procès-verbaux arriérés seront rédigés par les rédacteurs des deux Conseils ». Zélée, mais imprudente, elle ajouta « en gras » (corps du procèsverbal) « En vertu de la loi du 3 brumaire an IV »... Par ailleurs, ce même volume offrait la délicate «construction » de la séance du 9 thermidor,

(21) Les années 1960 indiquaient les départements contemporains et non les départements de 1790 ou de l'an II. C'est ainsi que le département de Paris subit une « double peine » : rebaptisé par les éditeurs « département de la Seine », il devient introuvable en 1968 ! Nous ne dirons rien des localisations révolutionnaires dans le Tarn-et-Garonne ou la Meurthe-et-Moselle.

(22) Archives Parlementaires, t. XCIII, 1982. 
pour laquelle un «traitement » extraordinaire fut finalement réservé ${ }^{23}$. Il apparaît avec clarté que les « rédacteurs postérieurs » de ce procès-verbal, le premier d'une longue série, sont tous députés du Conseil des Cinq-Cents, tous « réacteurs » notoires et aptes à mettre en valeur les enjeux et points de fracture d'une «thermidorialité », alors que la «centralité législative » demeure le «pivot central du gouvernement », jusqu'à la séparation de la Convention nationale.

\section{Dire et faire les Archives Parlementaires : le procès-verbal à la moulinette des Archives nationales}

Il faut rappeler que le procès-verbal, support traditionnel, présente les faits, non dans l'ordre où ils se sont déroulés mais dans celui où les secrétaires et rapporteurs déposaient les différents textes sur le bureau de l'Assemblée. Une première série de décrets permet de comprendre l'articulation entre les différents bureaux autour de la rédaction du procèsverbal officiel. La création du comité des Décrets, le 21 novembre 1789, ainsi que les décrets d'organisation des Archives nationales (entre le 4 et le 7 septembre 1790) mettent en place une navette pour la rédaction des procèsverbaux. La nomination de Camus, d'abord archiviste de l'Assemblée (14 août 1789) à la tête des Archives nationales - un député parmi les députés - , montre bien le souci politique de maîtriser les archives publiques. Pour la Convention, la section de la correspondance fait un premier tri avant de passer le relais à deux secrétaires-commis, Renvoisé et Aubusson, qui analysent la correspondance reçue pour le compte du président de l'Assemblée, puis sont chargés de préparer l'ordre du jour de la séance de la Convention ${ }^{24}$, dont en principe le procès-verbal devait être le reflet. Notons évidemment que la masse des adresses, pétitions, lettres de simples citoyens qui est publiée dans les Archives Parlementaires est forcément lacunaire et artificielle, puisque n'y sont présents que les documents retenus par l'Assemblée pour lecture, inscription au procès-verbal et, le plus souvent, mention honorable. Les documents non retenus ayant été détruits par ce bureau des procès-verbaux, bureau devenu un lieu stratégique si on en croit les décrets du comité des Décrets et procès-verbaux ${ }^{25}$ qui demandent

(23) Voir t. XCIII, op. cit., p. 372. Sur ces problèmes, voir Françoise BRUNEL, Thermidor. La chute de Robespierre, Bruxelles, Éditions Complexe, 1989.

(24) Martine SIN BlimA-BARRU, Le comité des décrets, procès-verbaux et archives. Mise en perspective d'un savoir administratif, thèse sous la direction de Jean-Clément MARTIN, Paris, Université Paris 1 Panthéon-Sorbonne, 2012.

(25) Un comité qui change de nom en fonction de l'évolution de ses attributions : commission de Surveillance de l'envoi des décrets (21 novembre 1789 - 6 juillet 1790) ; comité de la Surveillance 
au comité des Inspecteurs de la salle que les locaux soient gardés jour et nuit ou que soit interdite la présence des journalistes ou de toute personne étrangère au service ${ }^{26}$. Enfin, après une séance, les documents utilisés retournent au bureau des procès-verbaux afin de rédiger le procès-verbal de la séance ; celui-ci devant être relu en séance, adopté et signé par le président de la Convention et les secrétaires élus ${ }^{27}$.

À partir du 2 thermidor, on voit donc apparaître des signatures au procès-verbal, qui ne sont pas celles du président et des secrétaires alors en fonction. L'ampleur de cette modification est révélée par l'étude des Archives Parlementaires à partir de cette date jusqu'à la fin de frimaire an III, soit pendant cinq mois ${ }^{28}$ qui représentent cent cinquante-cinq séances de la Convention dont soixante-douze d'entre elles affichent un procès-verbal réécrit $(46,5 \%)$. Ces réécritures se font en vertu de trois lois distinctes : celles du 7 floréal an III $^{29}$ pour trente-cinq d'entre elles, du 3 fructidor an III ${ }^{30}$ pour douze séances et enfin du 3 brumaire an IV $^{31}$ pour vingt-cinq procès-verbaux. Pour la dernière référence, nul n'est besoin de souligner que nous sommes à la veille de la séparation de la Convention nationale, ce qui signifie que les procès-verbaux de thermidor an II sont réécrits sous le Directoire.

de l'envoi des lois et de la collation des décrets (6 juillet 1790 - 3 septembre 1791) ; comité des Décrets (26 octobre 1791 - 18 octobre 1792) ; comité des Décrets et procès-verbaux (18 octobre $1792-7$ fructidor an II) ; comité des Décrets, procès-verbaux et archives (7 fructidor an II - 3 brumaire an IV). (26) Voir aussi le travail d'Alain COHEN, Le comité des Inspecteurs de la Salle. Une institution originale au service de la Convention nationale (1792-1795), Paris, L'Harmattan, 2012.

(27) Pour la période étudiée, $42 \%$ des procès-verbaux ont été adoptés dans les séances suivantes. Le délai d'adoption est compris entre cinq jours (séance du 17 thermidor an II) et plus d'un mois (séance du 10 vendémiaire an III). Voir Archives Parlementaires, t. XCIV, p. 362 et t. C, p. 366.

(28) Cela concerne les tomes XCIII à CII déjà publiés et emprunte aux tomes CIII et CIV en cours de rédaction.

(29) Collection générale des décrets, Paris, Baudouin, vol. 61, à la date du 7 floréal an III.

(30) Ibidem, vol. 65, à la date du 3 fructidor an III.

(31) Ibid., vol. 67, à la date du 4 brumaire an IV. 


\section{Rédaction des procès-verbaux - du 2 thermidor an II au 30 frimaire an III}

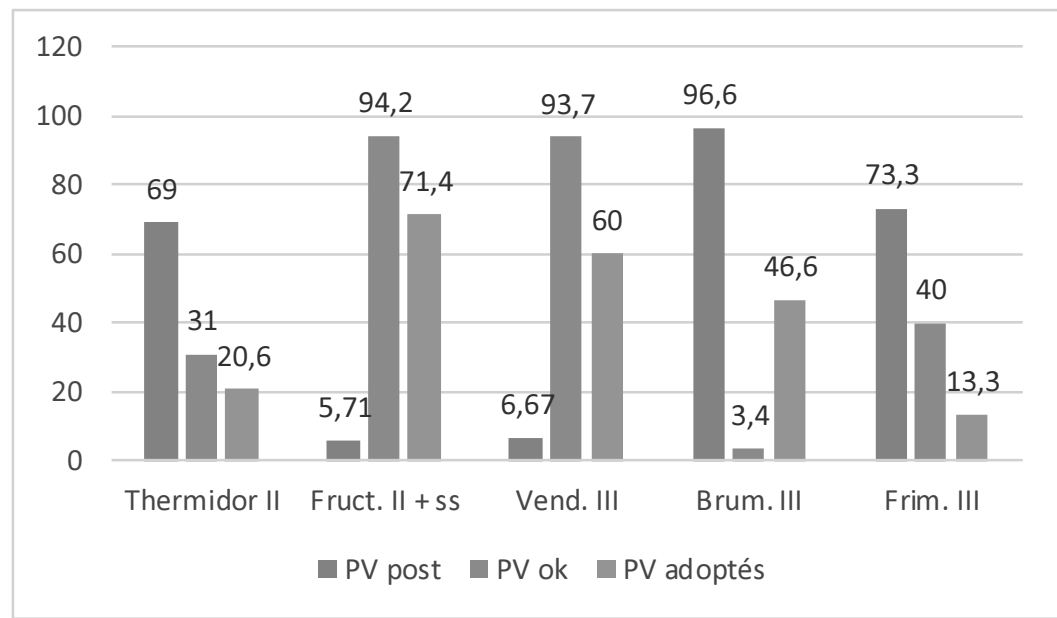

Les pourcentages ne se cumulent évidemment pas.

PV post : date de rédaction en vertu des lois de floréal, fructidor an III ou brumaire an IV.

PV ok : procès-verbaux rédigés et signés par le président et les secrétaires de la séance concernée.

PV adoptés : procès-verbaux adoptés selon mention dans les séances suivantes.

Une première lecture de ce graphique permet d'éliminer l'hypothèse d'une réécriture du procès-verbal selon ce que précisent ces différentes lois, à savoir qu'il s'agit de reprendre la rédaction des « procès-verbaux qui n'ont pu être signés » (loi du 7 floréal an III), « des séances en retard » (loi du 3 fructidor an III) ou encore « les procès-verbaux arriérés » (loi du 3 brumaire an IV). Brumaire an III contredit les termes de ces lois puisque $46,6 \%$ des procès-verbaux ont été adoptés dans les séances suivantes ce que réfute évidemment l'existence de $96,6 \%$ de procès-verbaux postérieurs ${ }^{32}$.

$\mathrm{Si}$ en thermidor, $69 \%$ des procès-verbaux sont réécrits en fonction de la loi du 3 brumaire an IV, les journées des 9 et 10 thermidor posent des questions. L'importance de ces journées est à prendre en compte ici en termes de validation politique par les conventionnels eux-mêmes : le 21 fructidor an II, seules les adresses et les pétitions de ces journées sont

(32) Ainsi le pourcentage de procès-verbaux adoptés dans les séances suivantes (42\%) que nous avions relevé dans notre comptage ne peut être affirmé. 
inscrites au procès-verbal, lequel est relu et adopté en séance ${ }^{33}$. Comme le souligne le journal Le Sans-culotte, «la rédaction n'est pas goûtée $»^{34}$; ainsi, Goupilleau (de Fontenay) obtient que deux conventionnels ${ }^{35}$ soient associés à la rédaction du procès-verbal afin que «le procès-verbal de cette séance mémorable [soit] une pièce pour l'histoire, que l'ordre des faits n'est pas moins nécessaire que l'exactitude ». Pénières, corroborant les propos de son collègue, ajoute enfin « que pour le rendre complet [le procès-verbal], il faut le lier à ce qui s'est passé le 8 , et le continuer jusqu'au jour où la permanence de la séance a été levée $»^{36}$.

Fructidor ${ }^{37}$ est un cas plus intéressant que celui de Thermidor, avec $94,2 \%$ des procès-verbaux signés par les conventionnels en poste et seulement deux journées qui affichent une réécriture du procès-verbal ${ }^{38}$. Le 12 fructidor où, faisant suite à un discours prononcé la veille par Tallien, Le Cointre (de Versailles) monte à la tribune pour dénoncer Billaud-Varenne, Collot d'Herbois et Barère du Comité de salut public, Vadier, Amar, Voulland et David du Comité de sûreté générale ; il fait lecture des vingtsix chefs d'accusation qu'il forme contre eux. Mais Le Cointre « avait mal choisi son moment et mal préparé son intervention et ne peut fournir aucune preuve », « ses vingt-six chefs d'accusation sont en fait des imputations de faiblesse [...] de la Convention $\gg^{39}$. Le lendemain, 13 fructidor, après une discussion assez houleuse, l'audition des pièces proposées par Le Cointre s'avérera impossible et les inculpations seront décrétées « fausses et calomnieuses », juste avant la levée de séance ${ }^{40}$.

Toutefois, il ne suffit pas de voir annoncée une reprise du procèsverbal pour s'interroger sur les creux et lacunes d'une source archivistique. Ainsi, la journée du 15 fructidor an II ( $\left(1^{\text {er }}\right.$ septembre 1794) est la journée des Archives Parlementaires où l'on note le plus grand nombre d'adresses envoyées à la Convention inscrites au procès-verbal ${ }^{41}$. On y retrouve mention d'environ cent cinquante adresses classées, ce qui prouve qu'elles

(33) Archives Parlementaires, t. XCVI, p. 322.

(34) Le Sans-Culotte, $\mathrm{n}^{\circ} 570$.

(35) Chénier et Charles Duval.

(36) Archives Parlementaires, t. XCVI, p. 322. Le 5 nivôse an III, la rédaction des procèsverbaux des 9, 10 et 11 thermidor est de nouveau ajournée. Voir Procès-verbal de la Convention, L II, $62-63$.

(37) Un mois de fructidor sur lequel nous avons aussi comptabilisé pour le graphique les cinq jours sans-culottides.

(38) En vertu de la loi du 3 brumaire an IV.

(39) Françoise BRUNEL, « L'épuration de la Convention nationale en l'an III », dans Michel VOVELLE (dir.), Le tournant de l'an III. Réaction et Terreur blanche dans la France révolutionnaire, Paris, CTHS, 1997, p. 20.

(40) Archives Parlementaires, t. XCVI, p. 125.

(41) Ibidem, t. XCVI, p. 161-165. 
sont bien passées dans les mains des secrétaires-commis, or ces adresses ne sont pas reproduites dans les Archives Parlementaires car elles sont introuvables dans la série $\mathrm{C}$ !

Pour vendémiaire an III, on note seulement deux procès-verbaux réécrits : celui du 29 vendémiaire, qui marque le début des discussions sur la procédure à prendre pour la mise en accusation de Carrier, et le décret de la Convention qui autorise les trois comités réunis à faire un rapport sur les députés détenus; et celui du 30 vendémiaire, où l'indigence du procès-verbal et le déficit d'archives de la série $\mathrm{C}$ ne permettent pas de tirer de conclusion - et nous laissent sur notre faim...

Brumaire an III nous interpelle fortement sur la réécriture des procèsverbaux : 96,6 \% d'entre eux sont réécrits, pour l'essentiel en vertu de la loi du 7 floréal an III. C'est d'abord à partir du 2, la présentation par Merlin (de Douai) au nom des trois comités réunis d'un «projet de loi sur les formes qui peuvent garantir la représentation nationale dans les accusations portées contre ses membres $»^{42}$, qui aboutit à la création de la commission des Vingt et un qui sera chargée d'examiner la conduite du représentant Carrier. Mais c'est aussi la séance - et non des moindres - du 22 brumaire, avec le décret sur la fermeture de la société des Jacobins, qui se fait sans débat, à l'unanimité moins une voix ${ }^{43}$.

Enfin, en frimaire, la Convention entre dans une brutale recomposition de son champ politique. Après avoir fermé le club des Jacobins et démantelé le réseau des sociétés populaires, elle choisit de punir Carrier et de réintégrer les députés exclus de juillet à octobre 1793 (les « $75 »)$. Une réintégration qui s'apprête à renverser les alliances et à fracturer le camp des « Thermidoriens $»^{44}$.

Ces réécritures posent la question des allers-retours entre les différents bureaux en charge des procès-verbaux et nous interroge à la fois sur la temporalité de ces modifications et sur les enjeux de la classification des archives. Si la Convention thermidorienne stabilise les entrelacements d'espaces administratifs et s'est enfin débarrassée de la tutelle du seul Comité de salut public, notons que les actions entreprises dans les derniers mois de son existence vont être l'objet d'enjeux politiques entre les institutions qui poursuivent leurs activités au-delà de brumaire an IV, comme les Archives nationales et les nouveaux ministères. Le versement des papiers

(42) Ibid., t. C, p. 129.

(43) Ibid., t. CI, p. 166-168.

(44) Jean-Clément MARTIN, Nouvelle histoire de la Révolution française, Paris Perrin, 2012, p. 475 . 
des comités et de l'Assemblée, entamé sous le Directoire, voit la destruction d'une partie très importante de l'ensemble documentaire ${ }^{45}$ qui peut s'expliquer par le changement de méthode de classement (de provenance et de typologie à une division thématique par ministère), ce qui n'exclut en rien un reclassement politique. Et si l'on tente de suivre la destinée des papiers partis du bureau des procès-verbaux à la fin de la Convention dans les différents états établis par les Archives nationales, on peut établir une fourchette chronologique dans laquelle ces fameux procès-verbaux auraient été de nouveau rédigés : entre brumaire an IV (novembre 1795) et l'an VII (1798-1799), pour l'essentiel, et au plus tard en brumaire an XIII (novembre 1804) ${ }^{46}$.

Ainsi, l'originalité d'une bonne moitié des journées des séances de la Convention nationale à partir de Thermidor an II est d'avoir été reconstruite par des rédacteurs des Conseils du Directoire dont bon nombre avaient intérêt à «oublier » ou, pour le moins, à présenter les journées dans le sens de la nouvelle orientation politique, celle où le peuple légitime la « révolution » de la Convention effectuée en Thermidor an II. On note ainsi deux grands moments de la construction des discours en fonction de la présentation du procès-verbal et des pièces annexées à celui-ci. $\mathrm{Au}$ lendemain de Thermidor, La Proclamation au peuple français est rapidement connue à Paris, en Seine-et-Oise et en Seine-et-Marne puis, par ondes concentriques, l'information parvient au grand bassin parisien et à ses marges, puis au reste du pays en particulier par l'intermédiaire du réseau des représentants en mission, souvent montagnards.

Si l'unanimité semble parfaite au lendemain de Thermidor autour de la condamnation de Robespierre, dès fructidor pourtant, avec la lecture par Louchet de l'adresse de la société populaire de Dijon ${ }^{47}$, s'annoncent les dénonciations du «modérantisme » et le fractionnement du discours politique. Se joue ensuite la construction de la signification de la rupture thermidorienne et l'Adresse au peuple français du 18 vendémiaire ${ }^{48}$ permet aux conventionnels de stabiliser les hésitations des dernières semaines.

(45) Martine Sin Blima-BARRU, op. cit., p. 30 et 55.

(46) AN, AB/VI/1 : État des Archives nationales au 1 $1^{\text {er }}$ prairial de l'an V (20 mai 1797) établi par Camus ; État des Archives de la République française rédigé au $1^{\text {er }}$ prairial de l'an VII (20 mai 1799) par Coru-Sarthe ; État des Archives nationales du 30 frimaire an XIII (21 décembre 1804) établi à l'attention du nouvel archiviste Daunou. Par la suite, le déménagement des archives à l'Hôtel de Soubise (1809) sera l'occasion pour Daunou de transformer l'ancienne série E en série C et de centrer la nouvelle classification autour de la loi, rétrogradant l'importance des procès-verbaux désormais sans enjeu politique.

(47) Archives Parlementaires, t. XCVI, p. 283-284.

(48) Ibidem, t. XCIX, p. 30-32. 
Cette adresse qui se répand dans le pays et occupe des séances importantes de lecture d'adresses permet de convaincre la Convention qu'elle restera le centre de ralliement de tous les bons citoyens, enfin débarrassés des « buveurs de sang ». Si l'on reprend le graphique précédent, on voit bien que la fracture principale de la période est brumaire an III et non thermidor an II. Ainsi, brumaire construit autour des accusations portées contre Carrier et la fin des Jacobins, une représentation de l'Assemblée dans laquelle la Convention se reconnaît d'autant mieux, que les adresses et pétitions reçues renvoient son propre discours. Ainsi s'invente un discours thermidorien relevant d'une même création discursive de la Terreur ${ }^{49}$, dont le reflet dans les Archives Parlementaires, façonné en amont, est confirmé a posteriori par la réécriture des procès-verbaux.

\section{La séance du 3 frimaire an III à l'Assemblée et l'appel nominal contre Carrier : quand le procès-verbal nous mène en bateau...}

Dans ces réécritures, certaines journées des Archives Parlementaires sont à regarder de plus près. La séance du 3 frimaire an III (23 novembre 1794), dont l'ironie veut qu'elle soit présidée par le très « terroriste » et très « réacteur » Legendre (de Paris) ${ }^{50}$, constitue une importante journée dans le tome CII et conclut onze sessions pendant lesquelles a été discutée l'affaire Carrier.

Avec dix-neuf affaires inscrites au procès-verbal, ce qui est peu compte tenu de l'appel nominal ${ }^{51}$, la séance débute comme à l'ordinaire par la lecture des adresses : toutes « remercient la Convention nationale de les avoir éclairés par les principes contenus dans l'Adresse aux Français, d'avoir comprimé les continuateurs des triumvirs en fermant leur repaire, de maintenir à l'ordre du jour sur les vertus républicaines [et] l'engagent à mériter de plus en plus en restant à son poste, en domptant à la fois les ennemis du dedans et du dehors, l'estime et l'attachement de tous les bons citoyens $»^{52}$. Si on ne peut dégager dans cette liste de logique géographique,

(49) Annie JouRdAN, «La journée du 5 septembre 1793. La terreur a-t-elle été mise à l'ordre du jour ? »; Jean-Clément MARTIN, « Dénombrer les victimes de la Terreur. La Vendée et au-delà », dans Michel BIARD, Hervé LEUwERS (dir.), Visages de la Terreur, Paris Armand Colin, 2014, p. 45-60, p. 155-166. Voir Jean-Clément MARTIN, Violence et Révolution. Essai sur la naissance d'un mythe national, Paris Seuil, 2006 ; id., Nouvelle histoire de la Révolution française, op. cit., $3^{\mathrm{e}}$ partie.

(50) Une séance commencée à $11 \mathrm{~h}$ du matin et terminée entre $2 \mathrm{~h} 30$ et $4 \mathrm{~h}$ (selon les sources) du lendemain matin.

(51) À titre de comparaison, on a une moyenne de trente-neuf affaires par jour pour le tome CII.

(52) Archives Parlementaires, t. CII p. 63. 
on peut s'interroger sur la chronologie d'arrivée de ces adresses sur le bureau de l'Assemblée. Outre cinq adresses non datées, trois le sont du 16 brumaire ; quant aux autres, elles sont antérieures au 10 brumaire et ont déjà plus de trois semaines ; une adresse des citoyens de Lons-leSaulnier (Jura), convoqués en assemblée générale par les représentants Besson, Sevestre, Foucher du Cher et Pelletier, est même datée du 8 vendémiaire an III ! Cette dernière exalte le bonheur retrouvé de tous les citoyens depuis la mort du tyran et souligne que «ce changement étonnant c'est l'ouvrage de la représentation nationale ${ }^{53}$. Si on compare la datation de ces adresses, on note dans les journées précédentes des adresses beaucoup plus tardives, de fin brumaire pour l'essentiel, dont l'enthousiasme à l'annonce de la fermeture des Jacobins, est rehaussé par le défilé de plusieurs sections parisiennes à la barre le $1^{\text {er }}$ frimaire ${ }^{54}$. On assiste ainsi à une extraordinaire manifestation d'unanimité conformiste. On peut donc légitimement s'interroger sur le tri ! Cela d'autant plus qu'on pointera dans les dernières lectures d'adresses avant la discussion sur Carrier, une lettre de Goupilleau (de Montaigu) rendant compte de sa mission dans le Gard, l'Hérault, l'Aveyron et le Vaucluse, qui concerne les suites de l'affaire de Bédoin, mais aussi une lettre non datée de la Maison commune de Dijon ${ }^{55}$, qui rappelle toute l'horreur qu'a suscitée la « fameuse » adresse de Dijon lue en séance le 19 fructidor précédent ${ }^{56}$ et qui dénonce les soutiens de Carrier comme «échantillon de la queue de Robespierre ${ }^{57}$. L'ordre du procès-verbal témoigne par l'exemple de la rétractation de Dijon et par les accusations portées contre Maignet, soit de la mise en place d'une orientation préalable au vote, soit d'une réorganisation du procès-verbal qui anticipe la conclusion de l'appel.

Dans ce contexte, le discours de Carrier $^{58}$, qui développe une argumentation essentiellement politique ${ }^{59}$ et pose la question de la légitimité de la violence révolutionnaire et le procès fait à la Révolution, devient inaudible pour ces thermidoriens «vertueux et probes ». La défense des idéaux de l'an II qu'assume Carrier se fracasse sur la construction en cours d'une nouvelle orientation politique et est de fait presque passée sous silence dans le procès-verbal et les journaux. Cette

(53) Ibidem, p. 69-70.

(54) Ibid., p. 19.

(55) Ibid., p. 73-74 et p. 78-79. La lettre de Goupilleau est datée du 18 brumaire.

(56) Moniteur, XXI, 691-692 ; Archives Parlementaires, t. XCVI, p. 283-284.

(57) Journal de la Montagne, $\mathrm{n}^{\circ} 129$.

(58) Un discours dont il fut difficile pour le comité des Inspecteurs de la salle de prendre la décision de l'impression, voir Alain COHEN, op. cit., p. 167-168.

(59) Archives Parlementaires, t. CII, p. 93-99. 
lacune des sources nous a ainsi décidé à reproduire in extenso le discours de Carrier, en sortant de notre protocole habituel, ce qui n'est pas sans poser de nouvelles questions sur l'établissement du corpus.

De la même façon, pose problème la retranscription de l'appel nominal demandant : "Y a-t-il lieu à accusation contre le représentant Carrier? » Si le président Legendre (de Paris) précise bien que les députés peuvent voter par oui ou non ${ }^{60}$, il proclame à la fin d'un vote qui dure environ 7 heures, que « sur 500 membres présents, 498 votèrent pour le décret d'accusation sans condition et 2 avec condition ${ }^{61}$. Or un examen détaillé des résultats fait apparaître 497 votants ( $65 \%$ de présents), auxquels il faut d'ailleurs soustraire quatre votants officiellement inscrits au procèsverbal alors qu'ils n'ont pu être présents sur les bancs de l'Assemblée ${ }^{62}$. Deux conventionnels, Dabray et Fleury sont notés absents sans motif, alors qu'ils ont été arrêtés avec les girondins l'année précédente ! Quatre autres députés font parvenir à la Convention leur vote en dehors de l'appel nominal (Poultier, Vadier, Ehrmann et Loiseau) ${ }^{63}$, mais seul le vote d'Erhmann sera comptabilisé, en précisant que « ce n'est pas une maladie politique » qui l'a tenu éloigné de l'Assemblée ${ }^{64}$. La question des nombreux absents (271 députés) est soulevée à plusieurs reprises, et l'impression de l'appel nominal peut être une nouvelle fois interprétée comme un « contrôle des troupes », à l'instar de l'appel nominal des 15-17 juin 1793, « pour constater quels sont les représentants du peuple qui sont à leur poste ». Il s'agit de mettre en place une procédure disciplinaire dans un contexte politique particulièrement tendu et de sanctionner par la publicité des votes ${ }^{65}$.

Lors des appels nominaux contre Louis XVI, la Convention érigée en jury d'accusation et jury de jugement, a multiplié les catégories de répartition des votes. Lors de l'appel nominal du 13 avril 1793 (Y a-t-il lieu à accusation contre Marat ?), certains députés ont refusé de voter (Lakanal,

(60) Ibidem, p. 100.

(61) Appel nominal des 3 et 4 frimaire, l'an III de la République française une et indivisible, sur cette question : Y-a-t-il lieu à accusation contre le citoyen Carrier, représentant du peuple ? Imprimé par ordre de la Convention nationale, distribué à ses membres et envoyé aux départements et aux armées. A Paris, frimaire an III.

(62) Forest et Michet, députés de Rhône-et-Loire, sont décrétés d'arrestation depuis juillet 1793 et ne rentreront dans le sein de la Convention qu'à la faveur du décret du 18 frimaire an III. Chedaneau, député de la Charente-Inférieure et Bouchereau, député de l'Aisne, ont voté oui à l'appel nominal mais sont en réalité en mission. Voir Michel BIARD, Missionnaires de la République, Paris, CTHS, 2002 (réédition, Paris, Vendémiaire, 2015), p. 467 et 480.

(63) Procès-Verbal de la Convention Nationale, L, p. 82.

(64) AN, C 327 (2), pl. 1445, p. 2 et 3 ; Moniteur, XXII, 597.

(65) Anne SimONIN et Corinne GoMEZ-LE CHEVANTON, «L'appel nominal : une technique pour la démocratie extrême (1789-1795)», $A H R F, 2009$, n $^{\circ} 357$, p. 67-101. 
Thirion ou Cavaignac), d'autres s'abstenant à haute et intelligible voix (Charles Delacroix) ou votant « nul » (Poultier). Or, dans l'appel contre Carrier, on ne retrouve aucune de ces différenciations, pas plus qu'il n'est fait mention des abstentions. Ainsi, de tous les appels nominaux où une question est posée, l'appel contre Carrier est le seul pour lequel le non n'existe pas, alors même que l'unanimité est illusoire. Dans cette séance, ce sont alors les silences des débats et de l'appel qui permettent de comprendre la construction d'un outil sémantique et politique au service d'une nouvelle union du peuple et de la Convention.

La publication des Archives Parlementaires ne prétend nullement « restituer» les séances journalières de la Convention et ne prétend pas même, comme le souhaitaient les éditeurs de 1907, donner «dans un ordre inexact, le compte rendu exact des séances », encore moins épuiser la complexité du fonctionnement de l'Assemblée. Mais la série se veut aujourd'hui cohérente et, avec les pleins et les vides propres à une source archivistique contrôlée par le pouvoir, entend donner de précieux instantanés changeants des rapports de force à l'Assemblée et de l'ensemble de l'opinion publique nationale, dans ses bavardages et ses silences. Du « gigantesque remue-ménage » de la Révolution, écrit Michel Vovelle, il est « un reflet dans une source particulièrement riche, voire privilégiée », les adresses et pétitions que «rassemblent les volumes des Archives Parlementaires $\gg^{66}$. On ne saurait rendre plus bel hommage au travail effectué depuis un demi-siècle pour rendre ce corpus patrimonial digne des exigences actuelles de la recherche.

Corinne GOMEZ-LE CHEVANTON CNRS UMS 622

IHRF, 17 rue de la Sorbonne, 75005 Paris Paris 1 Panthéon-Sorbonne - CNRS corinne.gomez.lechevanton@gmail.com

Françoise BRUNEL

Université Paris 1 Panthéon-Sorbonne IHRF, 17 rue de la Sorbonne, 75005 Paris f.brunel2@wanadoo.fr

(66) Michel Vovelle, La découverte de la politique. Géopolitique de la Révolution française, Paris, La Découverte, 1993, p. 220. 